\title{
PREGNANCY IN PATIENTS WITH CHRONIC KIDNEY DISEASE
}

\author{
Ahmed Nagah Nour El Din, Haitham Mohammad Al Amir \\ Internal medicine department, Faculty of Medicine, Sohag university.
}

\section{The normal kidney in Pregnancy.}

Pregnancy affects essentially all aspects of kidney physiology. The orchestration of changes that occur is a physiologic feat. Kidney and systemic hemodynamics are marked by significant volume expansion and vasodilation. Glomerular filtration rate (GFR) increases $50 \%$ and renal plasma flow (RPF) increases up to $80 \%$ as compared with nonpregnant levels. Tubular function and handling of water and electrolytes are altered, leading to mild increases in proteinuria, glucosuria, lower serum osmolality, and reductions in serum sodium levels. The kidneys are larger during pregnancy because of fluid retention, and physiologic hydronephrosis is common. (1) Hydronephrosis during pregnancy occurs in $43 \%$ to $100 \%$ pregnant women, and it is more prevalent with advancing trimester.(2) Serial quantitative measurements by ultrasonography demonstrate that the maximal incidence of hydronephrosis is reached at 28 weeks, with a $63 \%$ overall incidence of hydronephrosis. The dilated collecting system can hold 200 to $300 \mathrm{~mL}$ of urine, leading to urinary stasis and a $40 \%$ increased risk for pyelonephritis in pregnant women with asymptomatic bacteriuria versus nonpregnant women.(3)

The kidneys increase in length $(1-2 \mathrm{~cm})$ and volume $(70 \%)$ with dilation of the calyces, renal pelvis and ureters, features which are more prominent on the right. There is hormonal dilatation of the systemic blood vessels, leading to a decrease in systemic vascular resistance and a physiological lowering of blood pressure, especially during the first and second trimesters. Thereafter, there is a steady return of blood pressure to prepregnancy levels until term.(4)

Plasma volume increases significantly, leading to a $30-50 \%$ increase in cardiac output and resulting in a steady increase in renal blood flow (50\% by the mid-second trimester). This increases the glomerular filtration rate (GFR) and gives rise to frequency and nocturia, resulting in decreased creatinine blood levels. The increased vascularity of the kidneys makes a renal biopsy beyond 32 weeks risky. Despite the increase in renal blood flow, the pressure within the glomerulus remains unaltered owing to compensatory effects on the afferent and efferent arteriole, but this only occurs in a normal kidney. Any pre-existing disease in the kidney will be accelerated, often with deterioration in renal function.(4)

Pregnancy also affects the renal tubules. There is decreased reabsorption of glucose and bicarbonate, leading to glycosuria and bicarbonaturia. Despite the loss of bicarbonate, there is a persistent alkalosis owing to an increased respiratory rate. There is hypercalciuria, although the risk of renal stones is not increased as inhibitors of stone formation also increase. It is also common to find mild proteinuria, $<300 \mathrm{mg} / \mathrm{d}$, which may remain up to 6 months postpartum. Isolated pyuria is common and disappears by 3 months postpartum. Serum sodium may decrease by $4-5 \mathrm{mEq} / \mathrm{L}$ and serum osmolality falls to a new osmotic setpoint of about $270 \mathrm{mOsm} / \mathrm{kg}$.(4)

Estimating glomerular filtration rate in pregnancy 
SOHAG MEDICAL JOURNAL

Vol. 23 No.1 Jan 2019
PREGNANCY IN PATIENTS WITH CHRONIC

Ahmed Nagah Nour El Din.et al

Due to these physiological shifts in the typical markers of CKD (serum creatinine and urine protein), defining CKD in pregnancy is difficult. In the nonpregnant state, the widely used Kidney Disease Outcomes Quality Initiative staging system broadly defines CKD. Commonly used formulae to estimate creatinine clearance or estimated GFR (eGFR) are inaccurate in pregnancy. This includes the Cockroft-Gault and Modification of Diet in Renal Disease formulae. The Cockroft-Gault overestimates GFR, while in preeclampsia, the Modification of Diet in Renal Disease formula underestimates GFR compared with 24-hour creatinine clearance. Twenty-four-hour urine collections for inulin or creatinine clearance remain the gold standard for GFR measurement, but are clinically impractical and rarely needed, hence our ongoing reliance on serum creatinine. The heterogeneous classification of CKD in pregnancy studies contributes to underrecognition and uncertainties around diagnosis.(5)

Estimating proteinuria in pregnancy

Proteinuria needs to be measured first to monitor pre-existing proteinuria, which may increase substantially during pregnancy. The protein-creatinine ratio (PCR) is the preferred method for quantitating proteinuria in the non-pregnant state (6)and its use is recommended to monitor pre-existing proteinuria during pregnancy. The second indication is for the diagnosis of pre-eclampsia, which is defined as the new onset of hypertension (blood pressure (BP) $>140 / 90 \mathrm{mmHg}$ ) and proteinuria ( $>300 \mathrm{mg}$ protein in a 24-hour urine collection) after 20 weeks' gestation.(7)Although there are limitations to 24-hour urine collections, the use of PCR for the diagnosis of pre-eclampsia has been controversial, with discordant conclusions compared with 24-hour urine collections.(8)

Epidemiology of CKD in pregnant populations

Few studies have explored the population prevalence of CKD in pregnancy. A Norwegian population study provides the best data, suggesting CKD affects $3.3 \%$ of pregnancies, with the prevalence of CKD stages 1, 2, and 3 being $2.4 \%, 0.8 \%$, and $0.1 \%$, respectively.(9) Early stage CKD can be difficult to define in pregnancy, and robust prevalence data are lacking. Advanced CKD (stage 3-5) is estimated to affect 1:150 women of reproductive age and 1:750 pregnancies.(10)

Primary renal diagnosis and outcomes

CKD etiology has less influence on outcomes than CKD stage, proteinuria, hypertension, and preeclampsia. An exception is systemic lupus erythematosus (SLE) nephritis: outcomes are poorer than for women with comparable renal impairment from other causes, particularly if associated with antiphospholipid antibodies.(11) Women with autosomal dominant polycystic kidney disease have an increased risk of preeclampsia and urinary tract infection. Hepatic cysts may enlarge in the estrogenic environment, and known cerebral aneurysms should be assessed prior to labor. There is also a risk of intracystic hemorrhage during labor, and large renal cysts should be monitored prior to and following delivery.(12)

Outcomes in other common nephropathies including IgA nephropathy do not consistently differ from same-stage CKD of other etiology,(13) although robust comparative data between primary diseases remain limited. Diabetic patients (particularly if poorly controlled) have increased rates of preeclampsia, fetal abnormalities, and fetal loss; proteinuric diabetic nephropathy may worsen in pregnancy, and the combination of diabetes, proteinuria, renal impairment, and hypertension is particularly difficult. These risks may be elevated in women with "overt" diabetic nephropathy compared with those 
with early stage disease.(14) Establishing good glycemic control and lowering the hemoglobin A1c preconception is important to improve maternal and neonatal outcomes, including reducing the risk of fetal malformation. There is some evidence that the risk of congenital abnormalities in diabetic women may be increased in the presence of nephropathy, underscoring the need for optimized glycemic control.(15)

Management of pregnancy in women with CKD

Preconception planning

Given the high rate of associated morbidity, women with CKD should have prepregnancy counseling early in their disease course. Contraceptive options should be explored if a delay in pregnancy is desirable, with clear explanation of the benefits of careful pregnancy planning. Key areas for discussion include an exploration of risks and outcomes, assessment of relevant risk factors; optimizing modifiable factors including control of primary renal disease and blood pressure (BP); implementation of drug changes; decisions regarding timing of conception; and development of a comprehensive pregnancy management plan. Genetic counseling should be offered to women with inheritable renal disease. As for all pregnant women, preconception and first trimester folic acid supplementation is recommended. In dialyzed women, 3-5 mg/day may be required, due to losses during dialysis.

Pregnant women with CKD benefit from specialized (tertiary or quaternary) multidisciplinary team management including obstetricians, obstetric physicians/nephrologists, anesthetists, dietetic, and midwifery input.(16) While the overall risk of pregnancy problems is increased in women with CKD, many women will have uncomplicated pregnancies and excellent outcomes, but still require frequent antenatal review, particularly monitoring maternal renal function and BP, and fetal growth and well-being.(17)

\section{Management of primary disease}

Women with glomerulonephritis including lupus nephritis require quiescent disease and stable immunosuppression for at least 6 months prior to conception. Immunosuppression should be adjusted and continued throughout pregnancy to abrogate risk of disease flare (Table 1), with recent reports of successful use of tacrolimus for SLE nephritis in pregnancy.(18) Women with SLE should have testing for anti-Ro and anti-La antibodies, which are associated with neonatal lupus and congenital heart block, and markers of antiphospholipid antibody syndrome, which may necessitate specific management in pregnancy.Hydroxychloroquine should be continued through pregnancy in $\mathrm{Ro}+\mathrm{La}+$ women.(19) Comorbid conditions require specific attention. Good diabetic control is important preconception and during pregnancy, and is associated with better outcomes.(20) Urine surveillance should occur regularly, with aggressive treatment of asymptomatic bacteriuria, particularly in women with reflux nephropathy, autosomal dominant polycystic kidney disease, and immunosuppression. Prophylactic antibiotics should be considered for the remainder of the pregnancy after a first episode of urinary tract infection, although this is not universally implemented.(21)

\section{Diagnosis of pregnancy}

Renal excretion of $\beta$-human chorionic gonadotropin ( $\beta$-hCG) produced by somatic cells is reduced in CKD. Urine pregnancy tests may be falsely positive. Serial blood $\beta$-hCG testing and pelvic ultrasound are useful to confirm pregnancy and determine accurate 
SOHAG MEDICAL JOURNAL

Vol. 23 No.1 Jan 2019
PREGNANCY IN PATIENTS WITH CHRONIC

Ahmed Nagah Nour El Din.et al

gestational age, rather than date of last menstrual cycle, as irregular cycles and anovulation are common in advanced CKD.(22)

\begin{tabular}{|c|c|c|}
\hline drug & Pregnancy effects & Recommendation in pregnancy \\
\hline Prednisolone & \begin{tabular}{lr||} 
Maternal hypertension \\
and & GDM \\
Risk of & thymal \\
hyperplasia and adrenal \\
suppression in neonate \\
with high doses
\end{tabular} & $\mid \begin{array}{l}\text { May be continued } \\
\text { Avoid prolonged high doses }\end{array}$ \\
\hline Azathioprine & $\begin{array}{l}\text { No teratogenicity } \\
\text { Unable to be activated } \\
\text { by fetal liver }\end{array}$ & May be continued \\
\hline Tacrolimus & \begin{tabular}{|lr} 
Potential increased risk \\
of & GDM \\
Risk of & transient \\
neonatal & renal \\
dysfunction & and \\
hyperkalemia & \\
\end{tabular} & $\begin{array}{l}\text { May be continued } \\
\text { Monitor and adjust levels } \\
\text { Early OGTT in pregnancy especially } \\
\text { if combined with prednisolone } \\
\text { Check neonatal biochemistry }\end{array}$ \\
\hline Cyclosporine A & $\begin{array}{l}\text { No teratogenicity } \\
\text { Associated r with } \\
\text { maternal hypertension } \\
\text { Reversible effect on } \\
\text { fetal lymphocytes }\end{array}$ & $\begin{array}{l}\text { May be continued } \\
\text { Monitor and adjust levels }\end{array}$ \\
\hline $\begin{array}{l}\text { Mycophenolate } \\
\text { mofetil }\end{array}$ & $\begin{array}{l}\text { Teratogenic } \\
\text { embryopathic } \\
\text { Multiple congenital } \\
\text { defects of ears, digits, } \\
\text { oral cavity } \\
\text { Increased pregnancy } \\
\text { loss }\end{array}$ & Cease 12 weeks prior to conception \\
\hline Cyclophosphamide & $\begin{array}{l}\text { Teratogenic in first } \\
\text { trimester } \\
\text { Affects ovarian function } \\
\text { and fertility }\end{array}$ & $\begin{array}{l}\text { Cease } 12 \text { weeks preconception } \\
\text { Use in later pregnancy if critical to } \\
\text { maternal life }\end{array}$ \\
\hline $\mid \begin{array}{l}\text { Sirolimus } \\
\text { Everolimus } \\
\text { (mTOR inhibitors) }\end{array}$ & \begin{tabular}{||lr} 
Animal studies & suggest \\
teratogenicity, effects \\
on bone and fetal \\
growth. Data remain \\
very limited
\end{tabular} & $\begin{array}{l}\text { Cease } 12 \text { weeks preconception due } \\
\text { to lack of data to support safe use }\end{array}$ \\
\hline
\end{tabular}

Table (1)

Bramham K, Lightstone L. Pre-pregnancy counseling for women with chronic kidney disease. J Nephrol. 2012;25:450-459. 
SOHAG MEDICAL JOURNAL

Vol. 23 No.1 Jan 2019
PREGNANCY IN PATIENTS WITH CHRONIC

Ahmed Nagah Nour El Din.et al

\section{Hypertension}

Controlling hypertension before and during pregnancy is essential. A meta-analysis by Bramham et al of nearly 800,000 pregnancies in non-CKD chronic hypertensive women demonstrated markedly increased risks of superimposed preeclampsia, cesarean section, preterm delivery, intensive care admission, and perinatal death. Hypertension is an additive risk factor at all stages of CKD.(23) There is insufficient evidence regarding BP targets and treatment-related modification of outcomes in hypertensive CKD pregnancies, although the National Institute for Health and Care Excellence guidelines recommend maintaining BP $<140 / 90 \mathrm{mmHg}$ in hypertensive women with CKD. Concerns regarding effects of lower BP on fetal growth were addressed in the CHIPS trial, where tighter control of diastolic BP reduced severe maternal hypertension with no significant fetal impact(24). However, caution should be used in extrapolating these findings to women with CKD owing to potential differences in vascular disease burden or placentation.(25) First-line medications commonly used for pregnancy hypertension include methyldopa, labetalol, and nifedipine. Antihypertensive management in pregnancy.(26) Angiotensinconverting enzyme (ACE) inhibitors and angiotensin receptor blockers are contraindicated due to intrauterine growth restriction, renal dysplasia, oligohydramnios, and fetal death.(27)

\section{Proteinuria and nephrotic syndrome}

Proteinuria is an important parameter for risk stratification of maternal CKD. The sensitivity and specificity of the spot protein/creatinine ratio is adequate to exclude significant proteinuria, and enable monitoring of existing proteinuria. However, in many centers, 24-hour urine collections remain the preferred test. Proteinuria is more likely to commence or worsen in pregnancy as CKD stage advances, and is probably independently associated with adverse pregnancy outcome.(28) Little data are available regarding prophylaxis against venous thromboembolism in women who develop nephrotic-range proteinuria or nephrotic syndrome in pregnancy. Given the prothrombotic risks of nephrotic syndrome and pregnancy, along with other risk factors including obesity and immobility, it may be appropriate to commence prophylaxis with low-molecular-weight heparin. Doses should be corrected for renal function in advanced CKD. If underlying glomerulonephritis is suspected, immunosuppression may be appropriate. Substantial edema may be managed with diuretics, with monitoring for oligohydramnios. Albumin infusion has also been used; however, the supporting evidence is insufficient and proteinuria may be paradoxically increased.(29)

\section{Pregnancy in dialyzed women}

Despite reduced fertility in women with advanced (stage 4-5) CKD undergoing dialysis, pregnancies still occur, presenting significant clinical challenges. Historically, high rates of fetal loss $(>50 \%)$ and generally poor outcomes reinforced clinical pessimism. However, since the late 1990s, with recognition that increased dialysis intensity improves outcomes, successful birth rates have risen to 70\%-90\%, although pregnancies remained complicated by hypertension $(50 \%-70 \%)$, preeclampsia $(18 \%-67 \%)$, polyhydramnios (up to $40 \%$ ), intrauterine growth restriction $(17 \%-77 \%)$, and prematurity and low birth weight $(50 \%-100 \%) .(30)$

Improvements in live birth, gestational age, and birth weight have been observed with intensive hemodialysis regimens, achieved via daily (or near-daily) treatments, longer treatment hours, or nocturnal dialysis.(31) 
SOHAG MEDICAL JOURNAL

Vol. 23 No.1 Jan 2019
PREGNANCY IN PATIENTS WITH CHRONIC

Ahmed Nagah Nour El Din.et al

The Italian Study Group of the Kidney and Pregnancy suggests daily nocturnal dialysis for women without residual renal clearance, aiming for at least 36 hours per week.(31) Long-hours dialysis achieves lower maternal urea levels, reducing fetal-osmotic diuresis and polyhydramnios, and avoiding rapid fluid shifts, uteroplacental hypoperfusion, and fetal distress. Treatment should aim for predialysis blood urea nitrogen levels $<50 \mathrm{mg} / \mathrm{dL}$ $(17.9 \mathrm{mmol} / \mathrm{L})$ or urea levels $<100 \mathrm{mg} / \mathrm{dL}(16.6 \mathrm{mmol} / \mathrm{L})$; women with residual renal function may require less intense dialysis to achieve these targets.(32) Hemodialysis management considerations include attention to predialysis biochemical parameters, frequent review of ideal weight, BP control, anticoagulation, diet modifications, and anemia management. The timing of initiating dialysis has prognostic significance because residual renal function is an important determinant of outcome. Data from the ANZDATA registry demonstrated superior live birth rates (91\% vs 63\%), but no difference in gestational age or birth weight, in women with advanced CKD who commence chronic dialysis (predominantly hemodialysis) during pregnancy, compared with women already receiving chronic dialysis at conception.(33)

\section{Pregnancy in transplant recipients}

Fertility is significantly increased after kidney transplantation, and the chances of successful birth are markedly increased compared with the dialysis cohort.(34) The ANZDATA registry has reported an overall live birth rate of $76 \%$ ( $97.8 \%$ for pregnancies reaching $>20$ weeks gestation), with most pregnancy losses occurring in the first trimester, and the rate of elective termination falling over time from $25 \%$ in the 1970 s to $3 \%$ in the 2000s.(35) In particular, preeclampsia occurs in $25 \%-35 \%$ of pregnancies, and growth restriction or preterm birth in 30\%-50\%; rates have been shown to be similar between transplanted and dialyzed women.

For women with stable and good allograft function, pregnancy does not appear to adversely impact long-term graft function or patient survival, although some women will have transient graft dysfunction or failure of creatinine to fall during pregnancy.(36)

Clinical management is largely similar to the CKD pregnancy cohort. Issues specific to transplanted women include determining appropriate timing of conception posttransplant, adjustment of immunosuppression, surveillance of allograft function, and vigilant monitoring for preeclampsia, rejection, and infection in the immunocompromised recipient. As in women with $\mathrm{CKD}$, distinguishing preeclampsia from chronic hypertension or graft rejection can present a diagnostic dilemma.

The optimal timing of pregnancy is not conclusively established by the available data, but must be balanced against the window of opportunity for childbearing. Effective contraception should be strongly recommended peri-transplantation; contraceptive options require careful consideration based on comorbidities and individual factors, however, intrauterine devices or combined barrier/hormonal methods are considered the safest option.(37) Pregnancy occurring within 1 year from transplantation may be safe, but other studies suggest a longer time posttransplant is favorable. The recommended interval of transplantation to pregnancy is 1-2 years, provided graft function is stable, hypertension controlled, and the immunological environment favorable with low risk of rejection or infection.(38)

Immunosuppressive therapy must be adjusted preconception in transplant and nontransplant patients. Graft function should be monitored for at least 3 months following immunosuppression changes, with reassessment of donor-specific antibodies in 
sensitized recipients. Most studies report a large number of immunosuppression combinations used in pregnancy, making subanalysis of immunosuppression and pregnancy outcomes difficult. Calcineurin inhibitors (tacrolimus or cyclosporine), prednis(ol)one, and azathioprine are most commonly used, and considered reasonably safe, without increased risk of congenital defects above the background population rate.(39) During pregnancy, blood levels of tacrolimus and cyclosporine may fall, necessitating surveillance and dose adjustment. Tacrolimus pharmacokinetics alter in pregnancy with increased unbound active fraction, although this is not routinely measured. Tacrolimus and prednis(ol)one have been associated with diabetes mellitus, but evidence for increased rates of gestational diabetes in the kidney transplant cohort is inconclusive. Mycophenolate mofetil is known to increase early pregnancy loss and birth defects, and should be ceased preconception. More data are needed to explore the safety of mammalian targets of rapamycin inhibitors in pregnancy, with some evidence of effect on fetal metabolism and growth; therefore, avoidance is preferable.(40)

\section{References}

(1) Dunlop W. Serial changes in renal hemodynamics during normal human pregnancy. $\mathrm{Br} \mathrm{J}$ Obstet Gynaecol. 1981;88(1):1-9.

(2)Faundes A, Bricola-Filho M, Pinto e Silva JL. Dilatation of the urinary tract during pregnancy: proposal of a curve of maximal caliceal diameter by gestational age. Am J Obstet Gynecol. 1998;178(5):1082-1086.

(3) Rasmussen PE, Nielson FR. Hydronephrosis in pregnancy: a literature survey. Eur J Obstet Gynecol Reprod Biol. 1988;27(3):249-259.

(4) Smith MC, Moran P, Ward MK, Davison JM. Assessment of glomerular filtration rate during pregnancy using the MDRD formula. $\mathrm{Br} \mathrm{J}$ Obstet Gynaecol 2008;115:109-112.

(5) Koetje PM, Spaan JJ, Kooman JP, Spaanderman ME, Peeters LL. Pregnancy reduces the accuracy of the estimated glomerular filtration rate based on Cockroft-Gault and MDRD formulas. Reprod Sci. 2011;18:456-462.

(6) Eknoyan G, Hostetter T, Bakris GL, et al. Proteinuria and other markers of chronic kidney disease: A position statement of the National Kidney Foundation (NKF) and the National Institute of Diabetes and Digestive and Kidney Diseases (NIDDK). Am J Kidney Dis 2003;2:617622
(7) American College of Obstetricians and Gynecologists. Diagnosis and management of preeclampsia and eclampsia. Int $\mathbf{J}$ Gynaecol Obstet 2002;77:67-75.

(8) Price CP, Newall RG, Boyd JC. Use of protein: Creatinine ratio measurements on random urine samples for prediction of significant proteinuria. A systematic review. Clin Chem 2005;51:1577-1586.

(9) Munkhaugen J, Lydersen S, Romundstad PR, Wideroe TE, Vikse BE, Hallan S. Kidney function and future risk for adverse pregnancy outcomes: a population-based study from HUNT II, Norway. Nephrol Dial Transplant. 2009;24:3744-3750.

(10) Coresh J, Selvin E, Stevens LA, et al. Prevalence of chronic kidney disease in the United States. JAMA. 2007;298:2038-2047.

(11) Smyth A, Oliveira GH, Lahr BD, Bailey KR, Norby SM, Garovic VD. A systematic review and meta-analysis of pregnancy outcomes in patients with systemic lupus erythematosus and lupus nephritis. CJASN. 2010;5:2060-2068.

(12) Piccoli GB, Pia A, Vigotti F, et al. Autosomal dominant polycystic kidney disease: what do we need to know for counselling? EMJ Neph. 2014;2(1):5160.

(13) Limardo M, Imbasciati E, Ravani P, et al. Pregnancy and progression of $\operatorname{IgA}$ nephropathy: results of an Italian 
multicenter study. Am J Kidney Dis. 2010;56:506-512.

(14) Piccoli GB, Clari R, Ghiotto S, et al. Type 1 diabetes, diabetic nephropathy, and pregnancy: a systematic review and meta-study. Rev Diabet Stud. 2013;10:6-26.

(15) Bell R, Glinianaia SV, Tennant PW, Bilous RW, Rankin J. Peri-conception hyperglycaemia and nephropathy are associated with risk of congenital anomaly in women with pre-existing diabetes: a population-based cohort study. Diabetologia. 2012;55:936-947.

(16) Bramham K, Lightstone L. Prepregnancy counseling for women with chronic kidney disease. J Nephrol. 2012;25:450-459.

(17) Wiles KS, Bramham K, Vais A, et al. Pre-pregnancy counselling for women with chronic kidney disease: a retrospective analysis of nine years' experience. BMC Nephrol. 2015;16:28.

(18) Webster P, Wardle A, Bramham K, Webster L, Nelson-Piercy C, Lightstone L. Tacrolimus is an effective treatment for lupus nephritis in pregnancy. Lupus. 2014;23:1192-1196.

(19) Stanhope TJ, White WM, Moder KG, Smyth A, Garovic VD. Obstetric nephrology: lupus and lupus nephritis in pregnancy. CJASN. 2012;7:20892099.

(20) Bramham K, Rajasingham D. Pregnancy in diabetes and kidney disease. J Ren Care. 2012;38(Suppl 1):78-89.

(21) Bramham K, Lightston L. Karoshi MNS, B-Lynch C, Keith L. A Textbook of Preconceptual Medicine and Management. Carlisle: Sapiens Publishing Ltd; 2012. Preconceptual counseling for women with chronic kidney disease.

(22) COG Practice Bulletin No 77: screening for fetal chromosomal abnormalities. Obstet Gynecol. 2007;109(1):217-227.

(23) Bramham K, Parnell B, Nelson-Piercy C, Seed PT, Poston L, Chappell LC. Chronic hypertension and pregnancy outcomes: systematic review and metaanalysis. BMJ. 2014;348:g2301.

(24) on Dadelszen P, Ornstein MP, Bull SB, Logan AG, Koren G, Magee LA. Fall in mean arterial pressure and fetal growth restriction in pregnancy hypertension: a meta-analysis. Lancet. 2000;355:87-92.

(25) SOMANZ [Internet] Guideline for the Management of Hypertensive Disorders of Pregnancy. 2014. [Accessed October 18, 2015].

(26) Cooper WO, Hernandez-Diaz S, Arbogast PG, et al. Major congenital malformations after first-trimester exposure to ACE inhibitors. NEJM. 2006;354:2443-2451.

(27) Li DK, Yang C, Andrade S, Tavares V, Ferber JR. Maternal exposure to angiotensin converting enzyme inhibitors in the first trimester and risk of malformations in offspring: a retrospective cohort study. BMJ. 2011;343:d5931.

(28) Nevis IF, Reitsma A, Dominic A, et al. Pregnancy outcomes in women with chronic kidney disease: a systematic review. CJASN. 2011;6:2587-2598.

(29) Piccoli GB, Cabiddu G, Attini R, et al. Pregnancy in CKD: questions and answers in a changing panorama. Best Pract Res Clin Obstet Gynaecol. 2015;29

(30) Saliem S, Patenaude V, Abenhaim H. Pregnancy outcomes among renal transplant recipients and patients with end-stage renal disease on dialysis. J Perinat Med. 2016;44(3):321-327.

(31) Cabiddu G, Castellino S, Gernone G, et al. Best practices on pregnancy on dialysis: the Italian Study Group on Kidney and Pregnancy. J Nephrol. 2015;28:279-288.

(32) Alkhunaizi A, Melamed N, Hladunewich MA. Pregnancy in advanced chronic kidney disease and end-stage renal disease. Curr Opin Nephrol Hypertens. 2015;24:252-259.

(33) Jesudason S, Grace BS, McDonald SP. Pregnancy outcomes according to dialysis commencing before or after 
conception in women with ESRD. CJASN. 2014;9:143-149.

(34) Piccoli GB, Cabiddu G, Daidone G, et al. The children of dialysis: live-born babies from on-dialysis mothers in Italy - an epidemiological perspective comparing dialysis, kidney transplantation and the overall population. Nephrol Dial Transplant. 2014;29:1578-1586.

(35) Wyld ML, Clayton PA, Kennedy S, Alexander SI, Chadban SJ. Pregnancy outcomes for kidney transplant recipients with transplantation as a child. JAMA. 2015;169:1-6.

(36) Bramham K, Nelson-Piercy C, Gao H, et al. Pregnancy in renal transplant recipients: a UK national cohort study. CJASN. 2013;8:290-298.

(37) KDIGO KDIGO clinical practice guideline for the care of kidney transplant recipients. Am J Transplant. 2009;9(Suppl 3):S1-S155.

(38) Josephson MA, McKay DB. Women and transplantation: fertility, sexuality, pregnancy, contraception. Adv Chronic Kidney Dis. 2013;20:433-440.

(39) Kim H, Jeong J, Yang W, et al. The optimal therapy of calcineurin inhibitors for pregnancy in kidney transplantation. Clin Transplant. 2015;29:142-148.

(40) Framarino-dei-Malatesta M, Derme M, Napoli A, et al. Placental, lipid, and glucidic effects of mammalian target of rapamycin inhibitors: impact on fetal growth and metabolic disorders during pregnancy after solid organ transplantation. Transplant Proc. 2014;46:2254-2258. 